PROFESIONALES Y HERRAMIENTAS PARA EL DESARROLLO LOCAL Y SUS SINERGIAS TERRITORIALES. EVALUACIÓN Y PROPUESTAS DE FUTURO IX Coloquio Nacional de Desarrollo Local del GTDL-AGE 

ANTONIO MARTÍNEZ PUCHE, XAVIER AMAT MONTESINOS, ISABEL SANCHO CARBONELL y DANIEL SANCHIZ CASTAÑO (EDS.)

\section{PROFESIONALES Y HERRAMIENTAS PARA EL DESARROLLO LOCAL Y SUS SINERGIAS TERRITORIALES. EVALUACIÓN Y PROPUESTAS DE FUTURO}

IX Coloquio Nacional de Desarrollo Local del GTDL-AGE

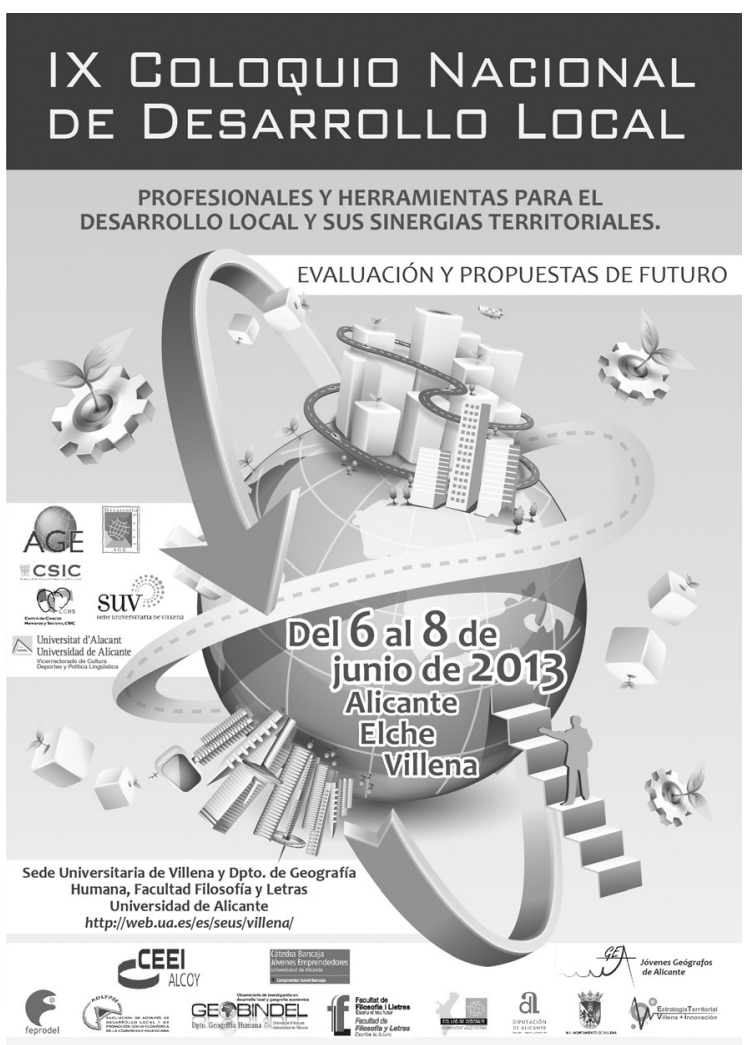


Este libro ha sido examinado y valorado por evaluadores ajenos a la Universidad de Alicante, con el fin de garantizar la calidad científica del mismo.

Publicacions de la Universitat d'Alacant

03690 Sant Vicent del Raspeig

Publicaciones@ua.es

http://publicaciones.ua.es

Telèfon: 965903480

(C) Antonio Martínez Puche, Xavier Amat Montesinos,

Isabel Sancho Carbonell y Daniel Sanchiz Castaño (eds.), 2016

(C) d'aquesta edició: Universitat d'Alacant

ISBN: 978-84-16724-00-0

Dipòsit legal: A 92-2016

Disseny de coberta: candela ink

Composició: Página Maestra (Miguel Ángel Sánchez Hernández)

Impressió i enquadernació: Guada Impresores

\section{unte \\ Unión de Editoriales
Universitarias Españolas \\ WWW.une.es
WWA}

Esta editorial es miembro de la UNE, cosa que garantiza la difusión y comercialización nacional y internacional de sus publicaciones.

Reservados todos los derechos. Cualquier forma de reproducción, distribución, comunicación pública o transformación de esta obra sólo puede ser realizada con la autorización de sus titulares, salvo excepción prevista por la ley. Diríjase a CEDRO (Centro Español de Derechos Repográficos, www.cedro.org) si necesita fotocopias o escanear algún fragmento de esta obra. 


\title{
EL CAMBIO DE LAS PRÁCTICAS DE DESARROLLO: PARTICIPACIÓN CIUDADANA, INNOVACIÓN SOCIAL Y DESARROLLO HUMANO SOSTENIBLE
}

\author{
Giedrè Šadeikaitè \\ Universidad de Alicante \\ g.sadeikaite@gmail.com
}

\section{RESUMEN}

Existe una disparidad entre el uso y la aplicación de la participación ciudadana como herramienta para aumentar la innovación social. Mediante la investigación integrada de literatura científica y de tres entrevistas, se analizará cómo la participación ciudadana mejora las capacidades y el poder para la toma de decisiones en el ámbito local, conlleva mayor innovación social ampliándose el desarrollo humano sostenible y cambia las prácticas de desarrollo. Se propone unir el marco de medición de la innovación social con el del desarrollo sostenible para poder captar el impacto de la innovación social en diferentes ámbitos.

Palabras claves: desarrollo humano, innovación social, participación.

THE CHANGE OF DEVELOPMENT PRACTICES: CIVIL PARTICIPATION, SOCIAL INNOVATION AND SUSTAINABLE HUMAN DEVELOPMENT

\section{Abstract}

The gap exists between the use and application of citizens' participation as the instrument for enhancing social innovation. Through integrated research of scientific literature and three interviews it is analysed how the participation improves capacities and power to take decisions in a locality, results in greater social innovation extending sustainable human development and changes practices of development. It is proposed to join the frame for measuring social innovation with the one of sustainable development to be able to capture the impact of social innovation in different environments.

Key words: human development, social innovation, participation. 


\section{INTRODUCCIÓN}

La participación ciudadana no es un concepto nuevo en el ámbito de desarrollo local. Son diferentes actores los que sin excepción enfatizan la importancia de la participación ciudadana en los procesos de desarrollo como herramienta obligatoria para la innovación social. No obstante, existe una diferencia enorme entre el uso del concepto y su aplicación real.

Este estudio a través de un análisis conjunto y sistemático de literatura científica y de tres entrevistas, con una experta de la organización internacional, un funcionario nacional y un empresario, intentará abordar cómo la participación ciudadana mejora las capacidades, las habilidades, la creatividad y el poder de tomar e influir en decisiones sobre las vidas de una comunidad, también cómo conlleva mayor innovación social aumentando el desarrollo humano sostenible y del mismo modo cómo cambia las prácticas de desarrollo.

Por consiguiente, el artículo trata de contribuir en la búsqueda de la aproximación del progreso y del impacto de la innovación social, siendo esencial para poder optimizar las prácticas de desarrollo. Se propone juntar el marco de medición de la innovación social existente con el marco del desarrollo sostenible que, por un lado, permita abordar los desafíos ambientales, económicos y socio-políticos y, por el otro, hacerlo de una manera interdisciplinaria considerando el impacto en el tiempo y espacio sin poner límites a cada ámbito.

\section{OBjetivos}

El objetivo principal es investigar cómo se puede perfeccionar las prácticas de desarrollo local a través de los procesos de innovación social mediante la participación ciudadana y la aumentación de sus capacidades. Es decir, la hipótesis que se propone es la siguiente: La participación ciudadana da lugar a una mayor innovación social y por lo tanto las prácticas de desarrollo local se pueden mejorar.

Otros tres objetivos para alcanzar el principal de este estudio son: El primero, analizar la importancia que varios actores en el ámbito del desarrollo local (concretamente a nivel internacional, nacional, y empresarial) dan a la participación ciudadana. El segundo trata de estudiar cómo la participación ciudadana influye en los procesos de innovación social. Y el tercero, investiga cómo la innovación social contribuye y cambia los procesos de desarrollo en las localidades.

\section{Metodología}

Este artículo trata de presentar y analizar las tres perspectivas de la participación ciudadana en relación a la innovación social para mejorar las prácticas de desarrollo local y sostenible desde el punto de vista de los expertos en desarrollo local, es decir, desde el ámbito internacional, nacional y empresarial. 
Los tres casos presentan un análisis cualitativo basado en las entrevistas no normalizadas y exhaustivas que fueron usadas para obtener unos datos primarios, aunque todas se basan en cinco preguntas principales, concretamente son:

1. Si la participación ciudadana es importante en el desarrollo internacional y los negocios, y en qué grado.

2. Cómo la participación civil influye o contribuye a los procesos de innovación social.

3. Cómo la innovación social influye y cambia los procesos y las prácticas de desarrollo.

4. De qué manera la inclusión de los ciudadanos contribuye al éxito y la sostenibilidad a largo plazo de los proyectos relacionados con el desarrollo.

5. Cómo las prácticas de desarrollo pueden ser mejoradas a través la participación de las comunidades locales y el aumento de sus capacidades y opciones.

Las entrevistas fueron realizadas a estos actores teniendo de éstos la información previa y el conocimiento de su compromiso en los proyectos de desarrollo para asegurar la fiabilidad de los resultados. Sin embargo, como la muestra de los casos no es representativa, la investigación es de baja validez externa y en consecuencia los resultados no obligatoriamente se pueden aplicar ampliamente a la población general. De hecho, la investigación pretende ofrecer un punto de partida para la búsqueda de la mejora de las prácticas de desarrollo local, analizando el estado actual de la innovación social a través de la participación ciudadana en la realidad, con énfasis especial en el desarrollo humano sostenible.

\section{LA PARTICIPACIÓN CIUDADANA Y DESARROLLO HUMANO SOSTENIBLE}

La participación ciudadana siempre fue considerada en los procesos de desarrollo. El desarrollo local puede ser calificado como las iniciativas "de la gente, por la gente y para la gente" (LINCOLN, 1863, párr. 3), a pesar que esta frase de Lincoln originalmente fue dicha sobre los gobiernos. La participación ciudadana, la planificación de la comunidad o cualquier otro término similar usado se ha convertido en un elemento inseparable de los gobiernos locales como una nueva dimensión de los procesos de la democracia moderna incluyendo actores diversos y usando técnicas diferentes para lograr objetivos diferentes (PRATCHETT, 1999: 616).

Por un lado, el que los ciudadanos se involucren en los procesos democráticos fomenta que la sociedad exprese su voz en la toma de decisiones que afectan sus vidas, y por otro, aborda la cuestión de la responsabilidad de los líderes y las instituciones políticas (INTERNATIONAL INSTITUTE FOR ENVIRONMENT AND DEVELOPMENT, 2001: 32). 
Actualmente, y a pesar del paso de los años, la importancia de la participación civil está obviamente creciendo. En la Conferencia de las Naciones Unidas sobre el Desarrollo Sostenible "RIO+20: el futuro que queremos", la Resolución final aprobada por la Asamblea General indica en numerosas ocasiones la importancia de la participación de la sociedad civil en los procesos de desarrollo sostenible, por ejemplo, el artículo 44 (UN, 2012) enfatiza "[...]el papel de la sociedad civil y la importancia de posibilitar que todos los miembros de la sociedad civil participen activamente en el desarrollo sostenible. [...] la mejora de la participación de la sociedad civil está supeditada, entre otras cosas, a la ampliación del acceso a la información y a la creación de capacidad de la sociedad civil y de un entorno propicio".

En algunas otras cláusulas se indica que "el pueblo debe tener oportunidades de influir en sus vidas" y en el futuro participando en la toma de decisiones y expresando sus preocupaciones fundamentales para el desarrollo sostenible, que puede ser logrado sólo con una amplia alianza de los gobiernos, la sociedad civil y el sector privado, es decir, todos trabajando juntos (UN, 2012: párr. 13 (Anexo)). También que la "gobernanza efectiva" a todos niveles es fundamental fortaleciendo el marco institucional para el desarrollo sostenible, que entre otras cosas (UN, 2012: párr. 76 (Anexo)) es necesario para fomentar la participación civil y su compromiso efectivo en la discusión internacional y en este sentido promover la transparencia y las asociaciones para ponerlo en práctica (UN, 2012: párr. 76 h (Anexo)). Lo que se muestra es el papel sustancial de la participación ciudadana en el desarrollo haciendo más enlaces con el desarrollo humano.

En la literatura de la economía del desarrollo, el término 'desarrollo humano' fue aceptado como "la expansión de las capacidades humanas, la ampliación de sus opciones, una mejora de las libertades y el cumplimiento de los derechos humanos" (FUKUDA-PARR \& KUMAR, 2011: xxi). En esta perspectiva, el aumento de los ingresos y la productividad deben ser vistos como los medios pero no los extremos del desarrollo, teniendo en cuenta que en el centro de desarrollo es (o debería ser) el avance del bienestar de la gente (ibíd.). Principalmente, el concepto enfatiza la cualidad y la distribución del crecimiento económico, su conexión con las vidas de la población y la sostenibilidad a largo plazo (FUKUDA-PARR \& KUMAR, 2011: 19).

El desarrollo humano fue formulado como un concepto holístico con cuatro pilares esenciales, en particular, la igualdad, la sostenibilidad, la productividad y el empoderamiento (FUKUDA-PARR \& KUMAR, 2011: 19). Su definición sigue diciendo que sólo las poblaciones que tienen capacidades, opciones y derechos a participar en la toma de decisiones pueden ser creativas e innovadoras iniciando los cambios que avancen en la calidad de sus vidas y las vidas en su comunidad, sin esperar que, por ejemplo, los gobiernos centrales o las comunidades internacionales actúen. 
Como ejemplo práctico se indica que en general las pequeñas iniciativas tienen más poder en resolver los problemas sociales, ambientales, culturales y económicos en una localidad. La razón principal del éxito normalmente es el capital humano local y las redes sociales, es decir, las personas locales son las que más saben sobre los desafíos de su entorno y por lo tanto, están en mejor posición de cambiar las iniciativas, instituciones y estructuras existentes que no son capaces de abórdalos. En otras palabras, el progreso humano es la precondición para que se produzca la innovación social y ésta tenga éxito y sostenibilidad a largo plazo en una localidad.

5. INNOVACIÓN SOCIALS: SUS DIMENSIONES Y DEFINICIONES PARA EL DESARROLLO LOCAL

El concepto de innovación social es relativamente nuevo como un tema predominante en las ciencias sociales, con sólo dos décadas de investigación intensa (MOULAERT, MARTINELLI, SWYNGEDOUW, \& GONZALEZ, 2005: 1969). Inicialmente la innovación social fue introducida en el ámbito de debate para la transformación de la sociedad per se. Es decir, en el área de política el énfasis fue puesto sobre el papel de la sociedad civil para el cambio social, mientras que en el debate económico se enfocó sobre el papel contra-cíclico de la economía social en la dinámica macroeconómica general (ibíd.).

Actualmente existen otras definiciones para la innovación social. La Revista de Innovación Social de Standford (2008: párr. 1) la describió en 2003 como "el proceso de la invención para la obtención de apoyo y aplicación de nuevas soluciones a las necesidades y problemas sociales". La nota importante fue puesta sobre la creación del dialogo entre los sectores público, privado y no gubernamental (ibíd.).

La Comisión Europea describe la innovación social como ideas, instituciones o maneras de trabajo nuevas para satisfacer las necesidades sociales más eficazmente que con los enfoques existentes (REEDER \& O'SULLIVAN, 2012: 7). Lo significativo es que permite iniciar los cambios importantes a coste relativamente más bajo (ibíd.).

Por otro lado, la OCDE se refiere a la innovación social como un cambio conceptual de los procesos y de los productos, también como cambios organizativos y en la financiación pudiéndose hacer frente a nuevas relaciones con las partes y territorios interesados (OECD, n.d.). Asimismo, otros términos que son usados describen la innovación social como nuevas system-changing ideas para resolver desafíos sociales, culturales, ambientales y económicos con beneficios para las poblaciones y el planeta que pueden tener lugar en todos los sectores (CENTRE FOR SOCIAL INNOVATION, 2004).

Sin embargo, desde su inicio como concepto científico la innovación social en el ámbito local y también como un marco analítico suele poseer los siguientes significados: 
1. RAÍCES COMUNES Y ESPECIFICIDADES NACIONALES que se refieren a los movimientos sociales a nivel local, regional o nacional con un objetivo de enfrentarse a los mecanismos de la explotación u opresión tanto actuales como potenciales en las políticas sociales y ambientales, empresa, cultura: inclusión versus exclusión social (MOULAERT ET AL., 2005: 1971);

2. ÉNFASIS EN LA COMUNIDAD, LA GOBERNANZA Y LA REPRODUCCIÓN - el objetivo fue incorporar la dimensión de lo "local" y la "comunidad" en las prácticas de la gobernanza como una manera más democrática que la del gobierno. Debe ser notado que la noción de la "comunidad" está muy fuertemente vinculado con el concepto de la "identidad local" cuando el fenómeno de la exclusión social está observado (ibíd.);

3. EL RESURGIMIENTO DE LA ECONOMÍA SOCIAL Y LA TRADICIÓN DE AUTOAYUDA pueden ser explicados como una compensación a la desintegración social en el estado de bienestar mediante las «redes de seguridad», es decir, autoayuda, ayuda mutua, asociaciones cívicas y religiosas. La noción aparece con una mayor importancia extrema en las áreas metropolitanas (ibíd.);

4. LAS TENSIONES ENTRE LAS INICIATIVAS COMUNITARIAS Y EL ESTADO CENTRAL ponen el énfasis en la gobernanza, orientada para destacar las características positivas de la democracia local y por lo tanto de las iniciativas de la comunidad y las iniciativas de base o grassroots initiatives. La razón fundamental es conseguir el control sobre el gobierno local para darle más poder en la provisión de los servicios sociales (MOULAERT ET AL., 2005: 1971-1972);

5. INNOVACIÓN SOCIAL VERSUS INSTITUCIONALIZACIÓN, se refiere a unas iniciativas más espontaneas, innovadoras y creativas con el objetivo de cambiar las corrientes prácticas desde abajo o buttom. Se plantea la cuestión sobre la sostenibilidad de las innovaciones sociales, es decir, las innovaciones que tuvieron más influencia espacial y un ciclo de sobrevivencia más largo, pero que a lo largo perdieron su originalidad y han sido más propensas a la burocratización y formalización (MOULAERT ET AL., 2005: 1972);

6. COMUNIDAD VERSUS SOCIEDAD, se ocupa de analizar concretamente las tenciones entre las comunidades, con frecuencia más pequeñas o más específicas, y la sociedad en general, donde las tendencias dominantes alejan las estrategias de desarrollo de esas comunidades (ibíd.);

7. LA INNOVACIÓN DE PRODUCTOS VERSUS LA INNOVACIÓN DE PROCESOS. En la discusión más contemporánea, la dimensión de los ,procesos“ en la innovación social ha sido más predominante que 
la innovación de productos, con referencias a las prácticas de buena gobernanza y el fortalecimiento de la capacidad o empowerment en las iniciativas sociales. Sin embargo, esta tendencia se está cambiando y la importancia de la innovación de productos se recupera con el crecimiento de las necesidades de población (ibíd.).

Principalmente, analizando temas de la innovación social el problema mayor es que, a pesar de su difuso uso, el concepto no es tratado en profundidad (MOULAERT ET AL., 2005: 1973). Efectivamente, como algunos ejemplos indican, cada institución tiene posibilidad de adaptar la definición según sus necesidades, al ámbito de su trabajo y a su territorio. Aun así, la cuestión de a quien sirven las innovaciones sociales quizá sea más importante analizando su capacidad, en particular, la innovación social que se da entre organizaciones no gubernamentales, el gobierno, o los miembros de las propias comunidades (JACOBS, 2013: párr. 2). Por un lado, con una definición estándar sería más fácil unificar las personas que trabajan dentro o fuera de temas similares, pero por otro lado, la definición haría más más difícil de unificar a las personas que trabajan en diferentes ámbitos (JACOBS, 2013: párr. 5).

En consecuencia, medir el impacto de la innovación social mediante la participación civil es extremadamente difícil y delicado por varias razones. Entre ellas: (1) la ceguera cultural cuando algunas prácticas están impuestas en una comunidad sin una adaptación propia; (2) la falta de un grupo de control, o en otras palabras, nos engañamos a nosotros mismos con que una intervención social funciona cuando en realidad no es así, debido involuntariamente a falsas medidas del impacto adoptadas; (3) el efecto Placebo que se produce en las intervenciones sociales y en algunas personas que creen que han recibido una intervención efectiva, pudiendo incluso hacer mejor que si la intervención busca ser realmente eficaz; y finalmente (4) los efectos de compensación deben ser equilibrados al cambio de un sistema social, como por ejemplo el caso de aquellas personas que ponen muy poco esfuerzo para encontrar un trabajo si el sistema social es muy seguro (ZOLLI, 2013).

\section{SÍNTESIS DE LAS EVIDENCIAS EMPÍRICAS}

Como ha sido descrito en la metodología, la parte empírica se basa en las cinco amplias y estrechamente relacionadas cuestiones de observación (1) ¿es importante la participación civil en el desarrollo?, (2) ¿cómo influye esta participación en los procesos de innovación social? y por consiguiente (3) ¿cómo cambia la innovación social los procesos del desarrollo? Asimismo se trata de avanzar en (4) ¿cómo contribuye la participación civil al éxito y la sostenibilidad a largo plazo de los proyectos de desarrollo? y finalmente (5) ¿cómo pueden ser mejoradas las prácticas de desarrollo a través de esta participación civil? 
Aunque en las tres entrevistas se aceptó que la participación ciudadana es significativa en el desarrollo internacional, las políticas nacionales y en los negocios, no en el mismo grado. Para las organizaciones internacionales y los empresarios, la implicación de las comunidades locales es de la mayor importancia en la planificación y ejecución de los proyectos de desarrollo. A la vista de una organización internacional el desarrollo es para la gente y sin ella, su trabajo 'no tendría sentido'. Igualmente, empresarios e instituciones internacionales promovían la movilización de las comunidades con una interacción permanente entre todos los actores para la creación conjunta del futuro.

No obstante, desde la perspectiva de la administración nacional el papel de la sociedad civil es mucho más pasivo, es decir, si los gobiernos nacionales expresan las necesidades de su población e inician la negociación, es razón suficiente para comenzar un proyecto. De hecho, si gobiernos y los políticos elegidos como representantes oficiales son responsables y transparentes, las iniciativas pueden tener mucho éxito sirviendo a las necesidades de población. Además, varios ejemplos fueron mencionados que en la fase de implementación, como que los gobiernos no facilitan ayuda o cooperación para otros socios, organizaciones internacionales y empresarios, dando lugar a retrasos, falta de financiación y a veces incluso el fracaso de los proyectos, perdiéndose inversiones materiales, dinero y esfuerzo humano. Así que no hay que olvidar que el factor humano y otros factores externos muchas veces tienen un impacto muy grande en los resultados finales.

En las respuestas obtenidas a la cuestión de cómo la participación ciudadana influye en los procesos de innovación social, de manera genérica se llegó al acuerdo en las tres entrevistas que la sociedad civil sirve como una fuente principal de ideas y soluciones innovadoras, que son un buen reflejo de las prioridades y necesidades de una comunidad local. Asimismo, la participación ciudadana es percibida como un instrumento vital para el empoderamiento de dichas poblaciones.

Según las respuestas de la perspectiva internacional, la innovación social de ninguna manera puede traerse a través de los expertos internacionales y por lo tanto es obligatorio 'ir y observar' qué y cómo se hace en un región para aprender sobre una comunidad y apoyarles. La práctica confirma que la innovación social se hace con el mínimo de los recursos financieros y materiales, impulsado por las personas locales o bottum-up y, a pesar que sea espontanea u organizada es sostenible a largo plazo. Aunque las subvenciones no son necesarias, cuando existen pueden servir como un buen estímulo. Además, desde el punto de la vista de la empresa, visión desde arriba abajo o top-down, combinada con liderazgo y acción local, permite aumentar los resultados y también funciona como aliciente para innovaciones. Por el contrario, las autoridades nacionales enfocan hacia la transferencia de conocimientos y know-how, me- 
diante consultas más que con innovaciones sociales per se sin dar una respuesta más explícita.

Además, los interlocutores no siempre podían indicar una diferencia clara entre la influencia de la innovación social a los procesos de desarrollo y la contribución de la participación ciudadana a la sostenibilidad de los proyectos de desarrollo a lo largo de los años. Por lo tanto, las respuestas a ambas preguntas estuvieron fuertemente interconectadas, es decir, sirvieron para complementarse entre sí.

Desde la perspectiva internacional, la participación ciudadana aumenta la inclusión de la sociedad en los proyectos de desarrollo, que muchas veces dependen de fondos públicos. No obstante, si los proyectos existentes amplifican la innovación social, como resultado de iniciativas creadas a través de innovaciones, éstas serán más sostenibles porque sirven para el progreso de la más gente. Los funcionarios nacionales y los empresarios presentan una aplicación de esta idea internacional en la práctica, poniendo el énfasis en la sostenibilidad de las iniciativas y el poder de incluir a los ciudadanos en la toma de las decisiones sobre sus vidas, con dos objetivos. En primer lugar hay que constatar que la mejora de su futuro está en sus manos y en su responsabilidad primaria, y en segundo que las iniciativas locales y el esfuerzo conjunto es necesario para avanzar. Asimismo, para la optimización del éxito es relevante incluir a la sociedad entendida de forma extensa en este proceso de innovación social, es decir, incluir a empresarios, industrias, artesanos, jóvenes, asociaciones y grupos marginales locales. Por consiguiente, la innovación social cambia los procesos de desarrollo mediante los ajustes sustanciales a diversas necesidades de la sociedad en múltiples regiones, religiones, y contextos socioculturales y políticos, y cuando la gente puede tomar decisiones sobre su propias vidas a través de su compromiso y su responsabilidad, con o sin apoyo y financiación externa, los proyectos tienen más oportunidad de sostenerse.

La discusión confirmó que la participación ciudadana tiene un papel sustancial en el desarrollo, fomentando los procesos de innovación social así como contribuyendo al éxito y la sostenibilidad de los proyectos de desarrollo. En consecuencia, la innovación social cambia las prácticas de desarrollo envolviendo la sociedad civil de un carácter activo en estos procesos, es decir, los ajustes importantes están implementados teniendo en cuenta las diferencias entre países, regiones, culturas, religiones, contextos económicos y políticos. Tras haber examinado esto, que de alguna manera abarca la discusión anterior, nos lleva a la última pregunta realizada a los interlocutores que fue sobre cómo las prácticas de desarrollo pueden ser mejoradas a través de la participación civil y la mejora de sus capacidades y opciones.

Desde la percepción del funcionario nacional, éste destacó que las poblaciones locales son significativas en el desarrollo y deben ser incluidas en estos procesos. Sin embargo, faltaba una clara propuesta de cómo el avance de las 
capacidades de los ciudadanos pueden mejorar las prácticas existentes. Por el contrario, el empresario y la experta internacional tomaron una posición más activa poniendo los ciudadanos en el centro del desarrollo, es decir, como la causa, el medio y el resultado del desarrollo. Sus respuestas indicaron algunos aspectos diferentes pero fueron al mismo tiempo complementarias. Desde la perspectiva internacional así como desde la empresa, se señaló que hay que promover más el desarrollo basado en cada área concreta, y encontrar los modos de incluir a las poblaciones locales de una manera activa a través de un dialogo abierto consiguiendo su compromiso. Principalmente, la participación de los ciudadanos debe ser una parte fundamental en cada iniciativa que la sociedad valore, elabore y disfrute de los resultados generados. La inclusión activa aumenta las capacidades, la creatividad y las opciones de una comunidad, y así en cada momento posterior su contribución crece en términos de calidad y cantidad.

Asimismo, del punto de vista de la empresa la implicación de los ciudadanos perfecciona la calidad de los procesos de desarrollo en general mediante la creación de compromisos para la cooperación y la interacción, que supone una mejora significante. Además, este patrón tiene un papel todavía más importante en el ámbito internacional debido a la diversidad cultural. Por lo tanto, es necesario establecer un ambiente donde el respeto y la confianza estén unidos como valor indiscutible, siendo cada voz igualmente importante y tratada de la misma manera. Por último hay que señalar que la implicación ciudadana también es extremadamente crucial en las comunidades pobres, y su participación sirve como instrumento que les enseña a no ser víctimas de la situación y pueden contribuir considerablemente a la mejora de sus vidas.

\section{LA MEDICIÓN DE LA INNOCACIÓN SOCIAL PARA EL CAMBIO}

Permitiendo a las personas del marco común tener un papel sustancial en los procesos de desarrollo, asumiendo responsabilidades y compromisos claros, se incrementaran sus capacidades y libertadas para influir y tomar decisiones con impacto sobre sus vidas. Así la participación ciudadana provoca los procesos de la innovación social que todavía más adelanta el envolvimiento de la sociedad local haciendo sus capacidades de innovar, su creatividad progresar para introducir los cambios necesarios para mejorar sus vidas. Por consiguiente, sus capacidades pone la base sólida que aumenta sus opciones, donde la comunidad local tenga más libertades tomar las decisiones, conozca y ejecute sus derechos, así como sus responsabilidades que al largo plazo resulta en crecimiento del capital humano local o en otras palabras se observa desarrollo humano sostenible.

Por otro lado, como el análisis empírico confirmó, la innovación social influye en los procesos de desarrollo humano que provocan los cambios de las prácticas de desarrollo, involucrando a las personas locales activamente lo 
que da lugar a ajustes decisivos con diferencias según la cultura, la religión, el contexto económico y político, y en general, según la localidad. Por lo tanto, la medición del progreso y del impacto de la innovación social es fundamental para poder perfeccionar las prácticas de desarrollo, en otras palabras, hay que aprender colectivamente lo que está sucediendo para que podamos incrementar esfuerzos (DEMONSTRATING VALUE, n.d.: párr. 2). Más allá, es necesario comunicar a los legisladores nacionales sobre la situación de la innovación social específica en su territorio para que la toma de las decisiones se base en buena información y se cree un enfoque a nivel nacional (MILDENBERGER, SCHIMITZ, \& BUND, 2013: p. 3).

La innovación social no siempre tiene una forma tangible, y muchas veces resulta transferible el conocimiento o know-how, la autoayuda, el aprendizaje, el liderazgo local particular, las nuevas prácticas y las instituciones para abordar los desafíos locales. Además, el significado de un contexto explícito es enorme porque las culturas diversas perciben y valoran los mismos asuntos de manera diferente según su educación, tradiciones, modelos de comportamiento, estilo de vida y otros factores que no pueden ser negados o no tenerse en cuenta.

Hay intentos para medir el impacto de la innovación social. Debe tenerse en cuenta que por un lado está la falta de una definición estándar para ella, lo hace difícil medir el impacto de las personas que trabajan en diferentes ambientes (JACOBS, 2013: párr. 5). Y por otro lado, el hecho de que la innovación social tenga un carácter muy particular dependiendo de cada localidad y cada comunidad, el marco para la medición debería ser bastante amplio y no limitante que podía ser aplicado y efectivamente usado en cada contexto. Por lo tanto, desde el punto de la vista de este estudio, el siguiente marco para la medición y evaluación de la innovación social (Figura 1) podría ser adoptado con el propósito de perfeccionar las prácticas existentes en el desarrollo.

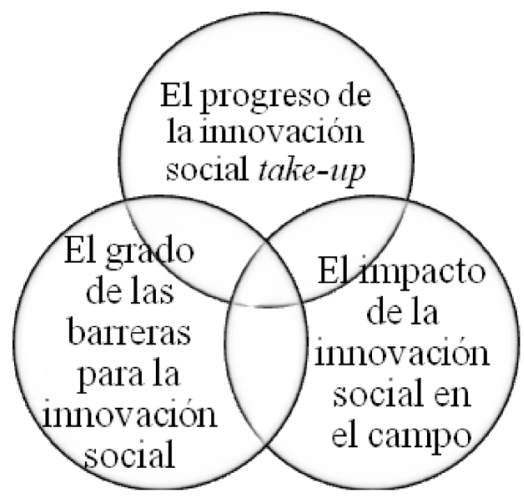

Figura 1. El marco para la medición y evaluación de la innovación social.

Fuente: REEDER \& O’SULLIVAN, 2012: 12. 
Los tres elementos principales del modelo se explican de la siguiente manera:

- EL PROGRESO DE LA INNOVACIÓN SOCIAL TAKE-UP se refiere a la medida en que las innovaciones sociales se utilizan en las sociedades y economías (REEDER \& O'SULLIVAN, 2012: 12);

- EL GRADO DE LAS BARRERAS PARA LA INNOVACIÓN SOCIAL explica la disponibilidad del liderazgo efectivo, los recursos financieros en cada etapa de la innovación, la experiencia y las habilidades humanas, la disposición y el voluntariado en una localidad y los estímulos para el cambio (ibíd.);

- EL IMPACTO DE LA INNOVACIÓN SOCIAL EN EL CAMPO mide el grado en que la innovación social está cambiando los resultados y la rentabilidad (ibíd.).

Si este marco se incorpora en el sistema de desarrollo sostenible (Figura 2), el esquema de la innovación social podía ser aplicado y usado en cada área del desarrollo sostenible, concretamente en los ámbitos social, económico y ambiental.

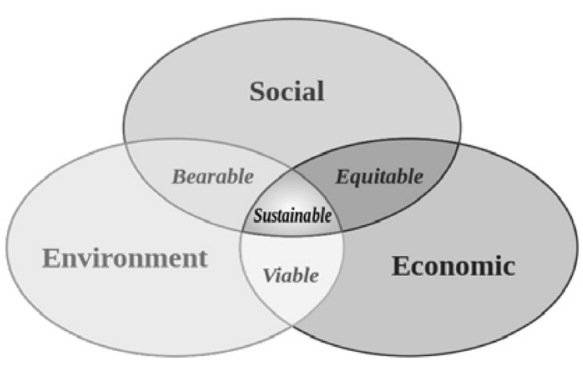

Figura 2: El marco de desarrollo sostenible.

Fuente: DRÉO, 2006.

Su aplicación es necesaria de tal manera que permita, en primer lugar, analizar el grado en que la innovación social es utilizada y manejada en una localidad concreta, acercándose a cualquier problema de una forma interdisciplinaria. Y en segundo lugar, hay que estudiar las barreras existentes que no permiten que el cambio suceda, ya sean barreras históricas, culturales, sociales, políticas, económicas o ambientales, para después buscar cómo las innovaciones sociales se produzcan.

Es de gran importancia que el análisis sea siempre de manera interdisciplinaria, viendo y analizando el impacto total para el desarrollo y sostenibilidad, e incluso la interrelación y el impacto de las decisiones entre las generaciones presentes y futuras y entretiempo y espacio. 
No obstante, las condiciones para que este modelo sea aplicable y funcional se basan en que la participación ciudadana de oportunidades a una comunidad de avanzar en sus capacidades y en iniciar los cambios necesarios para un progreso sostenible sin amenazar ni su presente y futuro en términos sociales, ambientes y económicos. Siendo una parte activa en estos procesos, la comunidad local tiene la posibilidad tomar decisiones mejor informadas y mejor consideradas, que provocan el cambio de paradigma en las prácticas de desarrollo local.

\section{Conclusiones}

El marco teórico así como el análisis empírico confirmó que la participación ciudadana tiene un papel importante en el desarrollo local. Sin embargo, basado en las evidencias empíricas, se observa que la extensión de la implicación de las personas del marco común no siempre es suficiente, y que en algunos casos los actores en el desarrollo abordan su participación de un modo pasivo. De hecho, es necesario crear consorcios con participación de todos los actores, es decir, locales, nacionales, internacionales y empresas, para que cualquier desventaja de un sector pueda ser abordada de una manera integrada y sistémica.

Facilitando y permitiendo la participación activa de las comunidades locales en las responsabilidades y los compromisos concretos, y también en la propiedad ilimitada de los resultados del desarrollo, se mejoran sus capacidades, habilidades y el poder de tomar e influir las decisiones sobre sus vidas. En consecuencia, lo anterior permite iniciar los cambios creativos e innovadores para mejorar sus vidas ampliando sus opciones y libertades que intensifican el desarrollo humano.

De la misma manera, la innovación social mediante la participación ciudadana con más poder, influencia, creatividad y capacidades, supone implicar a las personas locales todavía más activamente en los procesos de desarrollo que funcionan como catalizador para los cambios de las prácticas de desarrollo. Además, como el análisis empírico confirma, la inclusión de la comunidad local aumenta el éxito y la sostenibilidad a largo plazo de los proyectos e iniciativas de desarrollo.

Por consiguiente, el cálculo del progreso y del impacto de la innovación social es fundamental para poder mejorar las prácticas de desarrollo. Teniendo en cuenta el carácter muy particular de la innovación social, que depende en gran medida de cada localidad, comunidad y su cultura, el estudio propuso incorporar el marco de medición de la innovación social existente en el sistema del desarrollo sostenible. La anexión así permitirá el estudio cómo la innovación social está tratada localmente de forma interdisciplinaria, y las barreras existentes que no permiten que el cambio ocurra, para finalmente y a pesar de los obstáculos, se podrá observar como las innovaciones sociales acontecen y 
sobreviven. Asimismo, se podrá examinar como el crecimiento de la participación, la voluntad, la dedicación y el liderazgo local inicia sustantivos, eficaces, eficientes y sostenibles cambios afrontando los desafíos ambientales, económicos y sociopolíticos.

La limitación principal del modelo es el hecho de que éste no propone ningún sistema de medición concreto. Medición que en ningún caso ha sido objeto de este estudio, y que además requeriría de una investigación más detallada. Sin embargo, se cree que se hará un uso considerable de los indicadores y de los índices existentes y vínculos claros creados entre ellos con el fin de garantizar su compatibilidad, fiabilidad y aceptación más ampliamente.

La hipótesis de que la participación ciudadana da lugar a una mayor innovación social y por lo tanto las prácticas de desarrollo local se pueden mejorar está justificada y ha mostrado una fuerte solidez en su relación de causalidad. Sin embargo, lo que importa es la manera cómo la transcendencia y el impacto de la innovación social será capturados, medidos y comunicados para el cambio del paradigma actual de desarrollo.

BIBLIOGRAFÍA

CENTRE FOR SOCIAL INNOVATION. (2004). Social Innovation. http:// socialinnovation.ca/about/social-innovation, [consulta: 17/05/2013] DEMONSTRATING VALUE. (n.d.). Impact measurement can hinder social innovation. http://www.demonstratingvalue.org/blogs/impactmeasurement-can-hinder-social-innovation, [consulta: 19/05/2013]

DRÉO, J. (2006). Sustainable development. https://en.wikipedia.org/wiki/ File:Sustainable_development.svg, [consulta: 7/05/2013]

FUKUDA-PARR, S., \& KUMAR, A. K. S. (2011). Handbook of Human Development (Fourth edition.). New Delhi: Oxford University Press.

INTERNATIONAL INSTITUTE FOR ENVIRONMENT AND DEVELOPMENT. (2001). PLA Notes 40: Deliberative Democracy and Citizen Empowerment. UK: IIED.

JACOBS, M. (2013, May 14). Social innovation: lessons from Singapore, South Korea and Taiwan. The Guardian Social Enterprise Network. http://www. guardian.co.uk/social-enterprise-network/2013/may/14/social-innovationsingapore-south-korea-taiwan, [consulta: 20/05/2013]

LINCOLN, A. (1863). The Gettysburg Address by Abraham Lincoln, November 19, 1863. Abraham Lincoln Online. http://www.abrahamlincolnonline.org/ lincoln/speeches/gettysburg.htm, [consulta: 18/05/2013]

MILDENBERGER, G., SCHIMITZ, B., \& BUND, E. (2013, April 12). Towards Measuring Social Innovation: Some preliminary thoughts. Education, Center for Social Investment, University of Heidelberg. http://www.slideshare. net/SIeXchange/towards-measuring-social-innovation-some-preliminarythoughts, [consulta: 9/05/2013] 
MOULAERT, F., MARTINELLI, F., SWYNGEDOUW, E., \& GONZALEZ, S. (2005). Towards Alternative Model(s) of Local Innovation. Urban Studies, 42(11), p. 1969-1990.

OECD. (n.d.). LEED Forum on Social Innovations. http://www.oecd.org/cfe/ leed/leedforumonsocialinnovations.htm, [consulta: 16/05/2013]

PHILLS JR., J. A., DEIGLMEIER, K., \& MILLER, D. T. (2008). Rediscovering Social Innovation: Center for Social Innovation (CSI). Stanford Social Innovation Review, (Fall). http://csi.gsb.stanford.edu/rediscovering-socialinnovation, [consulta: 12/05/2013]

PRATCHETT, L. (1999). New fashions in public participation: towards greater democracy? Parliamentary Affairs, 52(4), p. 616-633.

REEDER, N., \& O'SULLIVAN, C. (2012). Strengthening social innovation in Europe: Journey to effective assessment and metrics. European Commission, Enterprise and Industry Magazine.

UN. (2012). Future We Want - Outcome document .: Sustainable Development Knowledge Platform. http://sustainabledevelopment.un.org/futurewewant. html, [consulta: 11/05/2013]

ZOLLI, A. (2013). Why We're So Bad At Measuring Impact, And How To Fix It. Co.Exist. http://www.fastcoexist.com/1681398/why-were-so-bad-atmeasuring-impact-and-how-to-fix-it, [consulta: 19/05/2013] 